\title{
ICD-11 PTSD and CPTSD: Implications for the rehabilitation of survivors of torture seeking international protection
}

\author{
Aisling Hearns' ${ }^{1}$, Philip Hyland ${ }^{2}$, Carin Benninger-Budel ${ }^{3}$ and Frederique \\ Vallières ${ }^{1}$
}

\section{Key points of interest}

- Evidence of construct validity for ICD 11 diagnoses of PTSD and CPTSD among a population of treatment-seeking survivors of torture and serious harm, seeking international protection in Ireland. Prevalence rates amongst this group, as measured by the International Trauma Questionnaire (ITQ), are high.

- Valid measurement of trauma-related disorders within populations of treatment-seeking survivors of torture has implications for diagnosis, assessment, treatment, and policy across rehabilitation centres for torture survivors globally.

\footnotetext{
Abstract

Introduction: Rates of torture are especially high among those seeking asylum, with global estimates of forced migrants having experienced torture exceeding $50 \%$. Torture

1) Trinity Centre for Global Health, University of Dublin.

2) School of Psychology, Maynooth University, Maynooth, Co Kildare, Ireland

3) OMCT International Secretariat. Correspondence to Aisling Hearns: hearnsai@tcd.ie
}

is the strongest predictor of PTSD amongst refugee populations. This study assesses the construct validity of the International Trauma Questionnaire (ITQ), a self-report measure of ICD-11 PTSD and Complex PTSD (CPTSD) symptoms, within a population of torture survivors seeking asylum in Ireland. It further explores whether probable rates of PTSD and CPTSD differ by sex and torture or serious harm status.

Methods: A secondary data analysis of 264 treatment-seeking asylum seekers and refugees who experienced torture or serious harm was conducted.

Findings: A confirmatory factor analysis found that a six-factor correlated model consisting of re-experiencing ( $\mathrm{Re})$, avoidance $(\mathrm{Av})$, threat ( $\mathrm{Th})$, affective dysregulation (AD), negative self-concept (NSC), and disturbed relationships (DR) provided optimal fit to the sample data. $32.4 \%$ of participants were diagnosed with PTSD and a further 36.9\% met criteria for CPTSD. Experiencing torture was significantly associated with higher odds of meeting criteria for PTSD. No significant differences between the sexes were found for rates of PTSD or CPTSD.

Discussion: Support for the construct validity of the ITQ was found among torture survivors actively seeking international protection in Europe. Given the high rates of PTSD and CPTSD found among torture survivors, 
rehabilitation centres for survivors of torture should consider CPTSD as part of their assessment and treatment programmes.

Keywords: Torture, ITQ, CPTSD, refugees, asylum-seekers

\section{Introduction}

According to the United Nations High Commissioner for Refugees, over 70.8 million people are forcibly displaced worldwide (UNHCR:Ireland, 2019). Often fleeing contexts of civil strife, war, and conflict, torture survivors are overrepresented within refugee populations (Baker, 1992)1 with torture acting as the strongest predictor of posttraumatic stress disorder (PTSD) amongst refugees (Jaranson \& Quiroga, 2011). Moreover, asylum seekers, compared to refugees, are at greater risk of PTSD, depression and anxiety (Toar, O'Brien, \& Fahey, 2009); and one Irish study found that $63 \%$ of asylum seekers had symptoms consistent with meeting diagnostic criteria for PTSD symptoms, compared to $21 \%$ of refugees (Crumlish \& Bracken, 2011).

The United Nations Convention Against Torture (UNCAT) is the leading document in the definition of torture and the rights of the survivors. Consistent with common definitions of torture, UNCAT highlights the elements of intentionality, a power-defenceless relationship, and infliction of pain or suffering, as characteristic of torture. While the Committee against Torture, the monitoring body of the UNCAT, has progressed in addressing violence by non-state actors, including sexual and gender-based violence against women (SGBV) over the past decades, the World Organisation Against Torture (OMCT) is concerned that the use of the UNCAT is still not equal among the sexes, with women often receiving less protection, prevention, or access to rehabilitation services (OMCT, 2019).
The psychological impact of torture is well-documented, and comparable to other forms of trauma inflicted by human design, including sexual and gender-based violence (SGBV) and other forms of intentional violence (Herman, 1992b). However, there remain several important gaps in the literature in terms of which diagnoses, assessments and treatment protocols are best suited to survivors of torture. Firstly, the lack of diagnostic terminology to effectively encapsulate the observed psychological responses of surviving torture has long been highlighted in the trauma literature (de C Williams \& van der Merwe, 2013). Herman (1992b), for example, observed that patients who had experienced torture were marked by changes in their personality related to their traumatic experience(s). Accordingly, Herman coined the term 'Complex PTSD' to describe what she believed to be a more accurate diagnosis for survivors of interpersonal violence and trauma, including torture (Herman, 1992b). Consistent with the torture experience, current literature suggests that Complex PTSD (CPTSD) symptoms are more likely to arise following exposure to severe, prolonged, or repeated trauma, particularly if interpersonal in nature (Herman, 1992b) or where there is a perception by the victim of being unable to escape, due to physical, psychological, or social constraints (Cloitre et al., 2011).

More recently, CPTSD was introduced to the diagnostic nomenclature in the latest edition of the World Health Organization's (WHO) $11^{\text {th }}$ version of the International Classification of Diseases (ICD-11). The ICD-11 now distinguishes between two trauma-based disorders: PTSD and CPTSD (WHO, 2018). One of the guiding principles for the ICD-11 in revising the trauma disorders was a focus on core symptoms such as to improve cross-cultural validity (Maercker et al., 2013). This 
is considered especially important as people from different cultures and sub-cultures have been found to experience trauma and express posttraumatic symptoms differently to people from Western countries (Marsella, 1996). In recent years, the empirical evidence supporting the reliability and validity of ICD-11 diagnoses of PTSD and CPTSD has accumulated across many cultural and trauma-exposed samples (Ben-Ezra et al., 2020). Furthermore, research conducted by Vallières et al. (2018) suggests a high prevalence of CPTSD among treatment-seeking refugee populations. Most recently, the presence of ICD-11 PTSD and CPTSD has been identified within a diverse group of treatment-seeking refugees resettled in Denmark, some of whom had experienced torture (Vang, Nielsen, Auning-Hansen, \& Elklit, 2019). However, this latter study focused purely on resettled refugees and did not include asylum seekers.

Secondly, there remains a need to identify the usefulness and validity of existing assessment tools to identify CPTSD among survivors of torture. The Istanbul Protocol, also known as "The Manual on Effective Investigation and Documentation of Torture and Other Cruel, Inhuman, or Degrading Treatment or Punishment", as the first set of international guidelines for documentation of torture and its consequences (OHCHR, 2004), states that any assessment should include a psychological assessment in addition to a physical one. However, the lack of consensus relating to appropriate diagnostic terms and measures for the psychological impact of torture has resulted in a gap in the credibility of psychological assessments with this population (Aarts, Van Wanrooij, Bloemen, \& Smid, 2019). Moreover, there is a recognised lack of valid and reliable measurement tools for assessing the psychological impact of torture in a cross-cultural population (Mollica \& Caspi-Yavin, 1991;
Pérez-Sales, 2018). More recently, the International Trauma Questionnaire (ITQ), developed in line with ICD-11 PTSD and CPTSD, has been found to have good reliability and validity across homogeneous samples of refugees (Vallières et al., 2018).

Finally, a gap has been noted in the ability to effectively evaluate torture treatment interventions (Basoglu, 2006). The UN Committee against Torture specifies that States should have mechanisms to oversee, monitor, evaluate, and report on implementation of Article 14 of UNCAT ('the right to rehabilitation') and that this should include the collection of data relating to survivors, their experiences, and the consequences of torture (Pietrzak, 2018). Accordingly, it is recommended that measures used for the evaluation of torture rehabilitation programmes also be valid, reliable and culturally appropriate (Jaranson \& Quiroga, 2011). Specifically, in relation to CPTSD, there is a need for "advances in the development, testing, and routine use of reliable assessment measures that include items representing the full range of symptoms that follow single or complex trauma-exposure" (Cloitre et al., 2011, pp. 623-624).

In view of the high rates of torture among refugees, including up to $50 \%$ of forced migrants in Ireland (Wilson, Hennessy, Dooley, Kelly, \& Ryan, 2013), understanding the psychological impact of torture as part of the forced migrant experience remains an important and underserved area. Specifically, understanding whether forced migrants who have experienced torture are at a higher risk of meeting a diagnosis of CPTSD. Given that 167 countries worldwide have ratified the UNCAT and, therefore, are legally subject to Article 14, which affirms that each state must provide for 'as full a rehabilitation as possible' for survivors of torture within their jurisdiction (UNCAT, 1984), the addition of CPTSD 
into the ICD-11 presents new opportunities for diagnosis, assessment, and treatment in relation to the severe and compounding trauma suffered by forced migrant populations. Accordingly, the current study put forward three specific objectives. First, we sought to determine the factor structure of ICD-11 PTSD and CPTSD amongst a sample of treatment-seeking asylum seekers and refugees in Ireland who had suffered torture or serious harm. Second, we sought to determine the point prevalence of CPTSD and PTSD, as assessed using the International Trauma Questionnaire, among this sample. Thirdly, we investigated whether refugees and asylum seekers who have experienced torture were more likely to meet the diagnostic criteria for CPTSD than PTSD, and whether any sex differences existed in probable rates of CPTSD and PTSD across the population tested due to the theorised link between SGBV in women and the development of CPTSD (Herman, 1992a).

\section{Methods}

\section{Participant Information}

Participants were 264 treatment-seeking asylum seekers and refugees assessed at intake into Spirasi's rehabilitation services. Spirasi is Ireland's national rehabilitation centre for survivors of torture and inhuman treatment, which provides an out-patient model of care to its service-users. Service-users are referred by a medical practitioner. Applications are then reviewed by a panel within Spirasi to establish if they fit the remit of the service. At the time of initial assessment, 93.9\% ( $\mathrm{n}=$ 248) of participants were 'Asylum Seekers', $3 \%(n=8)$ were 'Refugees', and a further $3 \%$ fell into the 'other category', which included those with Irish Citizenship or EU Citizens. Participants were predominantly male $(61.7 \%, \mathrm{n}=163)$ and $38.3 \%(\mathrm{n}=$
101) were female. The largely heterogeneous sample included participants from 29 different countries of origin, with Zimbabwe ( $\mathrm{n}=$ 47), Pakistan ( $\mathrm{n}=26$ ), and DR Congo ( $\mathrm{n}=$ 23) being the most frequent. The age range of participants varied from 14 to 69 years of age with a mean age of 34.34 years $(S D=8.9)$. For the purposes of this study, participants were divided into two categories. The first category is 'survivor of torture' as defined by the UN Convention of Torture Article 1. The second category is 'serious harm' within the meaning of Article 13(b) of the Qualification Directive. ${ }^{1}$ This division is based on the categories the rehabilitation centre, Spirasi, uses when reviewing applications to the service. Some service-users fell outside of the UNCAT criteria required to access the service but were accepted under the category of serious harm. 'Serious harm' indicates that the applicant had seriously harmed by a non-State actor i.e. in cases of SGBV. Using this categorisation, nearly two-thirds of participants $(72.3 \%, \mathrm{n}=$ 191) were identified as survivors of torture using UNCAT Article 1; when divided by sex, $57 \%(\mathrm{~N}=58)$ of females and $81.6 \%(\mathrm{~N}=$ 133) of males met the criteria for UNCAT. The remaining $27.3 \%(\mathrm{~N}=72)$ were identified under the category of 'serious harm' using Article 13(b) of the Qualification Directive; when divided by sex $42.6 \%(\mathrm{~N}=43)$ of females and $17.8 \%(\mathrm{~N}=29)$ of males met the criteria for serious harm. There was an exception of one participant who fell into neither category, who was categorised as a survivor

1 Council Directive 2004/83/EC: Serious harm consists of: (a) the death penalty or execution; or (b) torture or inhuman or degrading treatment or punishment of an applicant in the country of origin; or (c) serious and individual threat to a civilian's life or person by reason of indiscriminate violence in situations of international or internal armed conflict. 
of war.

\section{Ethical Aspects}

Ethical approval for the current study was sought and approved at the level of Spirasi's Board of Directors and Trinity's School of Psychology Research Ethics Committee ahead of carrying out data collection and analysis: Approval ID: SPREC112020-31. All participants had provided written informed consent and all data was processed and stored in relation to EU GDPR regulations.

\section{Materials}

Participants were assessed by a physician, a psychotherapist, and a psychosocial officer during their initial assessment within the rehabilitation service to establish whether they were survivors of torture or serious harm, based on the aforementioned criteria. The presence of ICD-11 PTSD and CPTSD were assessed using the International Trauma Questionnaire (ITQ) (Cloitre et al., 2018) (see Annex) wherein PTSD is characterised by three symptom clusters relating to reexperiencing $(\mathrm{Re})$, avoidance $(\mathrm{Av})$ and sense of threat (Th), and CPTSD is characterised by three additional symptom clusters: affect dysregulation (AD), negative self-concept (NSC), and disturbed relationships (DR). These latter three clusters are collectively referred to as Disturbances of Self-Organisation (DSO). All 18 items are answered on a fivepoint Likert scale with a range of 'Not at all' (0) to 'Extremely' (4). Diagnostic criteria for PTSD require a score of $\geq 2$ ('Moderately') for at least one of two symptoms in each cluster, along with endorsement of at least one functional impairment item, defined as a score $\geq 2$. CPTSD diagnosis requires that the PTSD criteria are met in addition to a score of $\geq 2$ ('Moderately') for at least one of two symptoms in each of the Disturbances of
Self-Organisation clusters, again along with endorsement of at least one functional impairment item, defined as a score $\geq 2$. While $41.7 \%(n=110)$ required the use of an interpreter for this assessment, $58.3 \%(n=154)$ carried out the assessment in English.

\section{Data Analysis}

The factor structure of the PTSD/CPTSD symptoms was assessed using confirmatory factor analysis (CFA) with four models being compared (Figure 1). Model 1 is a unidimensional model where all items load onto a single latent variable, CPTSD. Model 2 is a six-factor correlated model consisting of re-experiencing $(\mathrm{Re})$, avoidance $(\mathrm{Av})$, threat (Th), affect dysregulation (AD), negative selfconcept (NSC), and disturbed relationships (DR). Model 3 is a second-order variant of Model 2, which assumes that the correlations between the six first-order factors can be explained in terms of a single 'CPTSD' factor. Model 4 is a second-order model including two factors of 'PTSD' (explaining covariation between $\mathrm{Re}, \mathrm{Av}, \mathrm{Th}$ ) and DSO (explaining covariation between AD, NSC, and DR). All models were estimated in Mplus (Version 8), using the weighted least square mean and variance adjusted (WLSMV) estimator. Model fit was assessed using standard recommendations whereby acceptable model fit is indicated by a non-significant chi-square value; Comparative Fit Index (CFI) and TuckerLewis Index (TLI) values $\geq 0.90$; a root mean square error of approximation (RMSEA) value $\leq 0.08$; and a standardised root mean square residual $(\mathrm{SRMR})$ value $\leq 0.08$. To compare the four models, we examined differences in the RMSEA ( $\triangle$ RMSEA), as this fit index includes penalties for increasing model complexity, whereby $\triangle$ RMSEA values $>.015$ indicate a significant improvement in model fit. Internal reliability for PTSD and 
Figure 1. Loading patterns of models in confirmatory factor analysis

\section{Model 1: Unidimensional CPTSD}

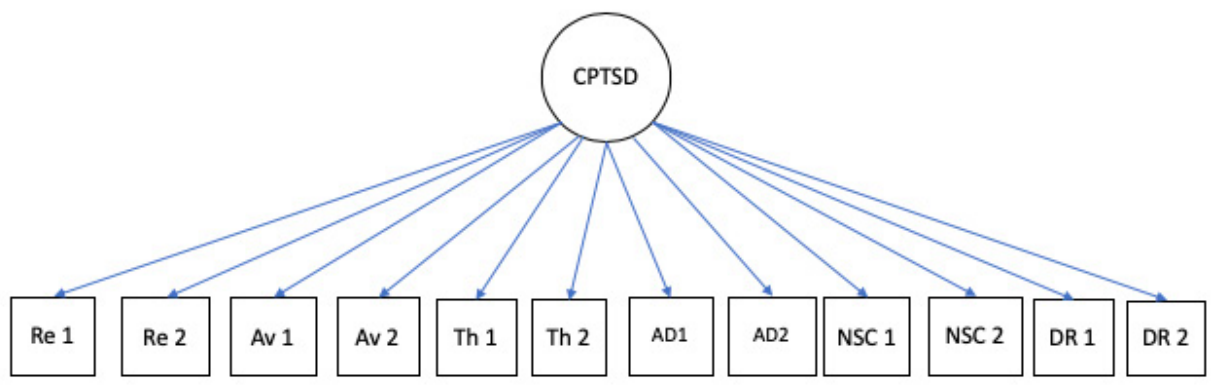

Model 2: Six-factor Correlated Model

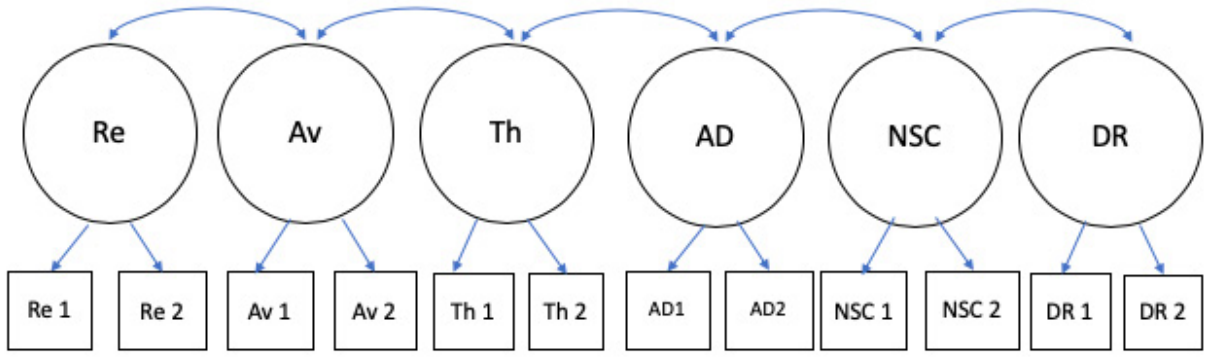

Model 3: Single-factor Second Order Model

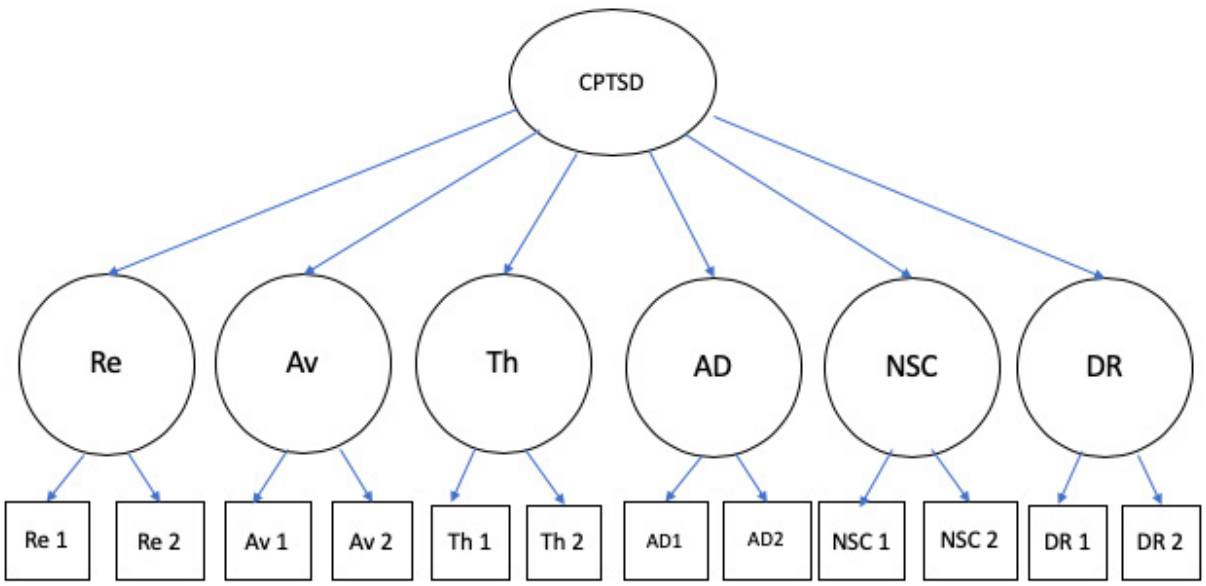




\section{Model 4: Two-factor Second Order Model}

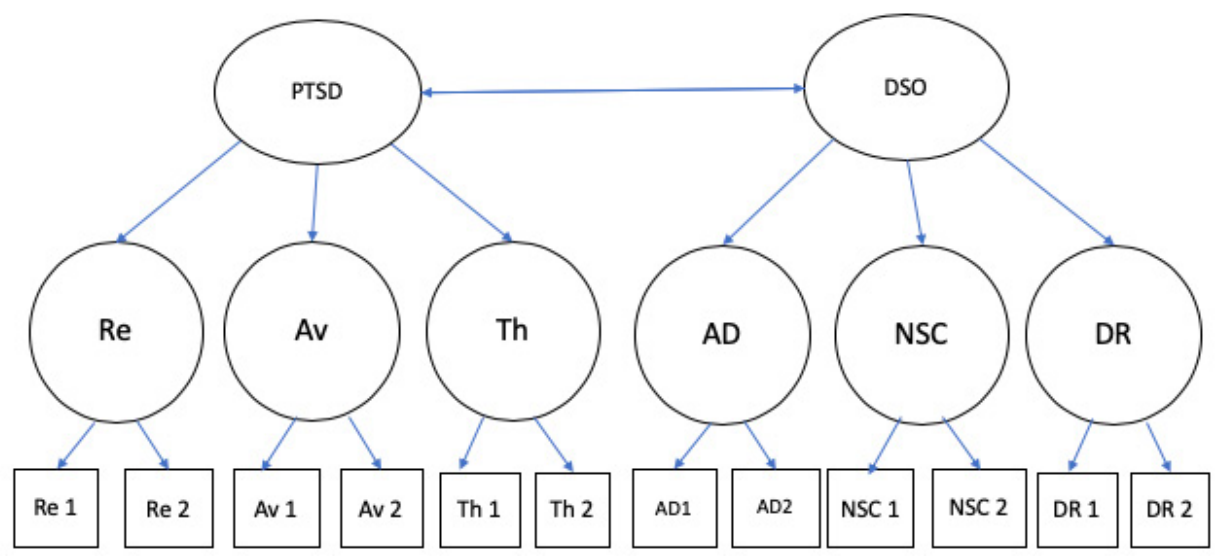

CPTSD was assessed using composite reliability scores. Prevalence rates for PTSD and CPTSD were calculated based on the best fitting model. Differences in diagnostic status across sex and UNCAT criteria were determined using a Pearson chi-square analysis in SPSS Statistics, Version 25.

\section{Results}

\section{Factor structure and internal reliability}

The CFA results are provided in full in Table 1. All models terminated normally. Of the four models tested, Model 1 was rejected as an unsatisfactory representation of the sample data given poor model fit indices. Models 2, 3, and 4 all produced good model fit. Model 2 had the lowest chi-square value, the highest CFI and TLI values, and the lowest RMSEA and SRMR values. Moreover, the $\triangle$ RMSEA values between Model 2 and Model 3 ( $\triangle$ RMSEA $=.035)$, and between Model 2 and Model $4(\Delta$ RMSEA $=.031)$ supported the statistical superiority of Model 2. Given that Model 2 was the best fit of the data, and consistent with the ICD-11 description of the symptom structure of CPTSD, it was deemed to the best model.

Table 2 presents the factor loadings for Model 2. All factor loadings were positive, significant $(\mathrm{p}<0.01)$ and greater than 0.45 . Table 3 presents the correlations between the six factors. All factor correlations were statistically significant $(\mathrm{p}<.01)$ and positive with correlations ranging from .24 to .86 .

\section{Diagnostic rates and descriptive statistics}

A total of 190 individuals $(72.0 \%)$ met the criteria for a probable diagnosis of either PTSD or CPTSD. Of these, $32.6 \%(n=86)$ were diagnosed with PTSD and 39.4\% $(n=$ 104) met criteria for CPTSD. There was no significant difference in the proportion of males and females who met criteria for PTSD $\left(34.9 \%\right.$ vs. $28.7 \%, \mathrm{x}^{2}(1)=1.111, \mathrm{p}=0.292$, $\mathrm{OR}=1.34,95 \% \mathrm{CI}=0.78,2.29)$ or $\mathrm{CPTSD}$ 
Table 1. Confirmatory Factor Analysis

\begin{tabular}{lllllll}
\hline & CFI & TLI & RMSEA & SRMR & $\chi^{2}$ & df \\
\hline & $>.90$ or .95 & $>.90$ or .95 & $<.08$ or .05 & $<.08$ & & \\
Model 1 & 0.883 & 0.857 & 0.119 & 0.073 & 254.9 & 54 \\
Mode1 2 & 0.999 & 0.998 & 0.016 & 0.029 & 41.483 & 39 \\
Mode1 3 & 0.981 & 0.974 & 0.051 & 0.044 & 80.513 & 48 \\
Mode1 4 & 0.984 & 0.978 & 0.047 & 0.042 & 74.217 & 47
\end{tabular}

( $35.6 \%$ vs. $45.5 \%, \mathrm{x}^{2}(1)=2.592, \mathrm{p}=0.107$, $\mathrm{OR}=0.66,95 \% \mathrm{CI}=0.39,1.096)($ see Table 4).

Those who met the UNCAT criteria for torture were significantly more likely to meet the criteria for PTSD (36.13\% vs. $22.2 \%, \mathrm{x}^{2}$ (1) $=4.621, p=0.032, O R=1.98 ; 95 \% \mathrm{CI}$ $=1.06,3.71$ ), but not for CPTSD $36.13 \%$ vs. $48.61 \%, \mathrm{x}^{2}(1)=3.410, \mathrm{p}=0.065, \mathrm{OR}=$ $0.60,95 \% \mathrm{CI}=0.35,1.04)$, than those that did not (see Table 4 )..

Under the category of 'serious harm' $20.9 \%$ $(\mathrm{N}=9)$ of females and $24.1 \%(\mathrm{~N}=7)$ of males received a diagnosis of PTSD. Under the category of torture, $34.48 \%(\mathrm{~N}=20)$ of females and $36.84 \%(\mathrm{~N}=49)$ of males received a diagnosis of PTSD. No significant differences were found between these groups. Prevalence rates of CPTSD with females who suffered 'serious harm' amounted to $55.81 \%(\mathrm{~N}=24)$, whereas males amounted to $37.93 \%(\mathrm{~N}=11)$. Prevalence rates for CPTSD with females who suffered torture amounted to $37.93 \%(\mathrm{~N}=22)$, whereas for males amounted to $35.34 \%$ (N $=47)$. No significant difference were found between these groups.

\section{Discussion}

The current study sought to determine the factor structure of the ICD-11 PTSD and CPTSD symptoms as measured by the ITQ amongst a sample of treatment-seeking asylum seekers and refugees in Ireland, the estimated prevalence rates of PTSD and CPTSD, and whether rates of PTSD and CPTSD varied depending on one's sex and history of torture or serious harm.

Findings from this study further support the validity of the ICD-11 PTSD and CPTSD constructs with asylum-seeking survivors of torture and serious harm. Specifically, our findings indicated that a six-factor model of the ITQ consistent with the description of CPTSD in the ICD-11 was an excellent representation of the sample data. Although three of the four models tested presented a good fit, this six-factor correlated model was deemed to be the best fitting model. This finding is consistent with earlier studies conducted by Kazlauskas et al. (2020) and Karatzias et al. (2016), who also showed goodness-of-fit with a correlated six-factor model of CPTSD, based on the proposed ICD-11 criteria using the ITQ. Conversely, Nickerson et al. (2016) found support for a two-factor higher-order model among refugees in Switzerland from a variety of different countries of origin using a number of adapted measures to assess symptoms related to ICD 11 PTSD and CPTSD, and Vallières et al. (2018) found support for a two-factor higher order model distinguishing between the symptoms of PTSD and CPTSD in treatment-seeking a sample of Syrian refugees in Lebanon, using the ITQ.

Our findings further lend support for the use of the ITQ as an appropriate diagnostic 
Table 2. Standardised factor loadings for the Two-Factor Second Order model

\begin{tabular}{|c|c|c|c|c|c|c|}
\hline & $\operatorname{Re}$ & $\mathrm{Av}$ & $\mathrm{Th}$ & $\mathrm{AD}$ & NSC & DR \\
\hline $\begin{array}{l}\text { Having upsetting dreams that replay part of the } \\
\text { experience or are clearly related to the experi- } \\
\text { ence? }\end{array}$ & 0.47 & & & & & \\
\hline $\begin{array}{l}\text { Having powerful images or memories that } \\
\text { sometimes come into your mind in which you } \\
\text { feel the experience is happening again in the } \\
\text { here and now }\end{array}$ & 0.87 & & & & & \\
\hline $\begin{array}{l}\text { Avoiding intern al reminders of the experience } \\
\text { (for example, thoughts, feelings, or physical sen- } \\
\text { sations)? }\end{array}$ & & 0.61 & & & & \\
\hline $\begin{array}{l}\text { Avoiding external reminders of the experience } \\
\text { (for example, people, places, conversations, } \\
\text { objects, activities, or situations)? }\end{array}$ & & 0.57 & & & & \\
\hline Being “super-alert", watchful, or on guard? & & & 0.79 & & & \\
\hline Feeling jumpy or easily startled? & & & 0.86 & & & \\
\hline $\begin{array}{l}\text { When I am upset, it takes me a long time to } \\
\text { calm down. }\end{array}$ & & & & 0.65 & & \\
\hline I feel numb or emotionally shut down. & & & & 0.66 & & \\
\hline I feel like a failure. & & & & & 0.80 & \\
\hline I feel worthless. & & & & & 0.84 & \\
\hline I feel distant or cut off from people. & & & & & & 0.86 \\
\hline $\begin{array}{l}\text { I find it hard to stay emotionally close to } \\
\text { people. }\end{array}$ & & & & & & 0.92 \\
\hline
\end{tabular}

Note. All factor loadings and factor correlations are statistically significant $(\mathrm{p}<.01)$.

Table 3. Correlations

\begin{tabular}{c|cccccc}
\hline & $\mathrm{Re}$ & $\mathrm{Av}$ & $\mathrm{Th}$ & $\mathrm{AD}$ & $\mathrm{NSC}$ & $\mathrm{DR}$ \\
\hline $\mathrm{Re}$ & 1.00 & & & & & \\
$\mathrm{Av}$ & 0.282 & 1.00 & & & & \\
$\mathrm{Th}$ & 0.537 & 0.502 & 1.00 & & & \\
$\mathrm{AD}$ & 0.863 & 0.328 & 0.695 & 1.00 & & \\
$\mathrm{NSC}$ & 0.640 & 0.244 & 0.521 & 0.748 & 1.00 & \\
$\mathrm{DR}$ & 0.693 & 0.261 & 0.511 & 0.856 & 0.780 & 1.00
\end{tabular}

Note. All factor loadings and factor correlations are statistically significant $(\mathrm{p}<.01)$. 
tool for assessing PTSD and CPTSD, as separate diagnoses, within a heterogeneous sample of asylum seekers and refugees who have experienced a range of extreme trauma. These findings have implications for the greater assessment, treatment, and support for survivors of torture accessing national torture rehabilitation centres. Medico-legal reports, used as evidence of torture for the purposes of international protection, are deemed an important part of the torture survivor's rehabilitation process. Aarts et al. (2019) analysed 97 medico-legal reports on traumatised asylum seekers in the Netherlands and found that the presence of physical evidence matching the asylum seeker's telling of events was positively associated with being granted asylum. However, the same association was not found for the psychological evidence presented, whereby the presence of psychological symptoms which matched the stated experience of the asylum seeker was not associated with being granted asylum. They concluded their study recommending a need to improve psychological and psychiatric assessments when documenting torture for the medico-legal process. The ITQ, as a validated measure of ICD-11 PTSD and CPTSD, therefore offers national torture rehabilitation centres a standardised tool that could be used to for psychological assessments carried out for Medico-Legal Reports, as part of the international protection process, and for the purposes of evaluation of treatment interventions offered by rehabilitation centres for survivors of torture. Having a standardised trauma assessment tool which has been validated with a diverse sample of survivors of torture seeking international protection offers a consistent platform for psychological assessment in the medico-legal process, which can

Table 4. Prevalence rates and relationships between ICD-11 PTSD and CPTSD and sex and torture/serious harm

\begin{tabular}{|c|c|c|c|c|c|}
\hline & ICD-11 PTSD & ICD-11 CPTSD & $P$ & OR & $95 \% C I$ \\
\hline Females & $\begin{array}{c}34.9 \% \\
(\mathrm{~N}=29)\end{array}$ & $\begin{array}{c}28.7 \% \\
(\mathrm{~N}=46)\end{array}$ & & & \\
\hline Males & $\begin{array}{c}35.6 \% \\
(\mathrm{~N}=57)\end{array}$ & $\begin{array}{c}45.5 \% \\
(\mathrm{~N}=58)\end{array}$ & & & \\
\hline $\mathrm{PTSD}^{\star}$ Sex & & & 0.292 & 1.34 & $0.78,2.29$ \\
\hline $\mathrm{CPTSD}^{\star}$ Sex & & & 0.107 & 0.66 & $0.39,1.09$ \\
\hline Survivors of torture & $\begin{array}{l}36.13 \% \\
(\mathrm{~N}=69)\end{array}$ & $\begin{array}{l}36.13 \% \\
(\mathrm{~N}=69)\end{array}$ & & & \\
\hline $\begin{array}{l}\text { Survivors of serious } \\
\text { harm }\end{array}$ & $\begin{array}{c}22.2 \% \\
(\mathrm{~N}=16)\end{array}$ & $\begin{array}{l}48.61 \% \\
(\mathrm{~N}=35)\end{array}$ & & & \\
\hline $\begin{array}{l}\text { PTSD`Torture/ } \\
\text { Serious Harm }\end{array}$ & & & 0.032 & 1.98 & $1.06,3.71$ \\
\hline $\begin{array}{l}\text { CPTSD }^{\star} \text { Torture/ } \\
\text { Serious Harm }\end{array}$ & & & 0.065 & 0.60 & $0.35,1.04$ \\
\hline
\end{tabular}


be used across global settings where international protection is sought.

The use of a common, valid measure could also contribute to generating more comparable outcomes across rehabilitation centres, and substantiate the effectiveness of the various treatments being used worldwide (Jaranson \& Quiroga, 2011). Specifically, while most rehabilitation centres worldwide for survivors of torture offer similar treatment models relating to therapeutic and psychosocial support, there is a lack of data available on how effective these treatment models are measured across a range of psychological outcomes. Of the few studies exploring the effectiveness of rehabilitation programmes on survivors of torture, high rates of psychopathology relating to PTSD, depression and anxiety have been recorded, with symptom prevalence in those who are not receiving treatment being shown to worsen (Lie, 2002) or remain stagnant (Vaage et al., 2010). Where studies do exist, they tend to focus almost exclusively on PTSD (Jaranson \& Quiroga, 2011) with little recognition or acknowledgement of the need to study other diagnoses, symptoms, functioning and impairment, including, and most notably, CPTSD, which our findings evidence is highly prevalent among a population of torture survivors seeking international protection in Ireland. Whilst it is worth noting the high prevalence rate of overall trauma diagnosis within this sample, i.e. $72 \%$, it is important to recognise that the ICD-11 presents PTSD and CPTSD as two distinct disorders, meaning that if an individual qualifies for a diagnosis of CPTSD, they cannot also be diagnosed with PTSD at the same time. Prevalence rates for PTSD and CPTSD in the current sample were $32.6 \%$ and $39.4 \%$ respectively.

Rates of PTSD and CPTSD among the current sample of treatment-seeking survivors of serious harm and torture were significantly higher than those reported in general population studies (Karatzias et al., 2019). This is not unexpected given the refugee experience is often marked by compounding instances of trauma throughout the migration journey (Crumlish \& Bracken, 2011; Wilson et al., 2013). It is worth noting however, that rates of both PTSD and CPTSD were higher in the current sample than those previously observed within other treatment-seeking refugee samples still living in a refugee camp (Vallières et al., 2018), but lower than that observed in a sample of refugees resettled in the host country (Vang et al., 2019), and among resettled refugees in Switzerland (Nickerson et al., 2016). Higher rates of PTSD and CPTSD identified in the current sample, may be further explained by the noted negative impact of the international protection process in Ireland on the mental health of asylum seekers (Crumlish \& Bracken, 2011). Notably, and as outlined in the United Nations Committee on the Elimination of Racial Discrimination, in their 2011 report entitled 'The Policy of Direct Provision', Ireland's inordinate delay in the processing of asylum seeker's applications and the final outcomes of their appeals and reviews, as well as poor living conditions characteristic of Direct Provision Centres, can suffer health and psychological problems that in certain cases lead to serious mental illness (UNCERD, 2011). Given that, in Ireland, up to $50 \%$ of forced migrants have suffered torture (Wilson et al., 2013), and that the physical and psychological impact of torture is often compounded by further trauma experienced during transit and on arrival in the host country, with the impact of post-transit trauma increasing over time (Beiser \& Hou, 2001), the impact of the host-country experience and the psychological impact of torture often present as intertwined. 
While a high rate of trauma-related disorders as a result of torture across both sexes is expected and has been supported by other studies (Ibrahim \& Hassan, 2017), our findings did not support significant sex differences for PTSD or CPTSD among this population of survivors of torture and serious harm. Indeed, possible sex differences in prevalence of ICD-11 PTSD and CPTSD are a subject of debate, with some studies reporting sex differences in PTSD and/or CPTSD (Shevlin et al., 2018) and others reporting no difference (Karatzias et al., 2017). Under the category of 'serious harm' our findings showed that women were more likely to meet a diagnosis for CPTSD (55.8\%) than men (37.9\%), but these differences failed to reach significant levels. This could be due to the lack of power to detect any systematic differences, given the small sample size available in the current study. Despite not reaching significant levels, these findings suggest that people who have faced interpersonal violence and disempowerment (e.g., SGBV) suffer a level of psychological effect equal to someone who is a survivor of torture at the hands of a State agent i.e. CPTSD. These similarities have been recognised by the UN Committee against Torture, the body monitoring UNCAT, in their interpretation of the definitions of torture and ill-treatment, by including gender-based forms of violence by non-state actors into its work. Specifically, in 2007, the UNCAT adopted 'General Comment 2' and stated in paragraph 18 that States are required to exercise due diligence to prevent, investigate, prosecute, punish and provide remedies for acts of non-state violence, including acts of gender-based violence such as rape, domestic violence, female genital mutilation, and trafficking and that otherwise "its officials should be considered as authors, complicit or otherwise responsible under the Convention for consenting to or acquiescing in such impermissible acts". The diagnostic levels of CPTSD among those who suffered serious harm found in this study's sample further supports a need for an inclusion of survivors of sexual and gender-based violence in the area of torture assessment and rehabilitation.

Taken together, findings of this study have important implications towards treatment modalities and best practice. Given the high prevalence of CPTSD within this population, traditional approaches for the treatment of PTSD may not be the most appropriate or effective among refugee or asylum-seeking populations. Treatment programmes for PTSD have been well-established, reviewed and agreed upon in academic and professional circles (Forbes, Bisson, Monson, \& Berliner, 2020). Patel, Williams, and Kellezi (2018) challenge the suitability of the likes of Eye Movement and Desensitisation Reprocessing and Cognitive Behavioural Therapy approaches to PTSD treatment when working with torture survivors. Cloitre et al. (2011) note that "there are few studies exploring adaptations of, or alternatives to, established PTSD treatments developed specifically for individuals with complex trauma histories and intended to target complex PTSD symptoms" (p. 616). Indeed, some of the treatment programmes developed for PTSD (i.e. Cognitive Behavioural Therapy) were shown to be less effective in those who had more complex trauma experiences (van der Kolk, Roth, Pelcovitz, Sunday, \& Spinazzola, 2005). Cloitre et al. (2011) also explored the recommended treatment modalities for Complex PTSD as opposed to PTSD and concluded that there was strong $(84 \%)$ consensus among experts that a phase-based approach, tailored to address relevant symptoms within the CPTSD criteria, is most appropriate.

Originally developed by Janet in 1925, the phase model approach today is most associ- 
ated with (Herman, 1992b), who advocated for this treatment model in her work with patients with Complex PTSD. Herman's phase model approach includes 1 . Safety-building, 2 . Grieving/Processing Trauma, and 3. Integration. A review of this approach nearly 20 years later has shown it continues to stand the test of time (Courtois, 2009). Whereas complexities emerge when considering appropriate treatment approaches to adult-onset CPTSD in refugee or war/genocide-exposed populations (Cloitre et al., 2011), a phase-model approach, with an emphasis on beginning with safety-building, which also takes into account the further complexities of family separation, cultural dislocation, and ongoing asylum-crises (Beltran \& Silove, 1999), is deemed most appropriate. This approach is further supported by a review by Nickerson, Bryant, Silove, and Steel (2011), who found a multimodal approach, which emphasises an initial safety-building/stabilisation phase, as more appropriate than PTSD treatment approaches for refugee populations. This approach is further endorsed by both the National Institute for Clinical Excellence (NICE) and the International Society for Traumatic Stress Studies (ISTSS) (Cloitre et al., 2011). Given the high prevalence of CPTSD within this population of torture survivors seeking international protection, the ICD 11 diagnosis of CPTSD, and its sibling diagnosis of PTSD, present opportunities for studies assessing the effectiveness of the phase model approach within torture rehabilitation centres.

The current study is not without limitations. Firstly, insufficiently powered analysis, due to the small sample size, may have resulted in the failure to detect meaningful sex differences. Secondly, the complexity of the international protection process in Ireland may have contributed to participants suffering additional distress (O'Connell, Duffy, \&
Crumlish, 2016), further compounding any pre-departure psychological distress. It must also be noted that the population in this study were treatment-seeking and therefore the prevalence rates are likely an overestimation of true prevalence rates of PTSD and CPTSD among asylum seekers and refugees who have experienced torture. Further to this, given that the ITQ was administered by several different Western white clinicians, human error and potential bias must be considered. Future research should seek to investigate the additional impact of the asylum-seeking process. In addition, a better understanding of the role of power-dynamics in torture, serious harm, and gender, as possible mechanisms for the development of CPTSD is required.

\section{Conclusion}

This is the first study to examine the prevalence of PTSD and CPTSD among a treatment-seeking sample of asylum seekers that have experienced torture. Our results suggest that, aligned to ICD-11, PTSD and CPTSD are common within this population group. The International Trauma Questionnaire offers a simple, valid measure with which to assess PTSD and CPTSD among this culturally diverse group. Sex differences were not found on significant levels of PTSD and CPTSD. These findings have implications for improving practice in the assessment and treatment of forced migrant populations who have experienced torture and serious harm in keeping with recommendations as laid out by the Istanbul Protocol (OHCHR, 2004) and bring to the fore a need for more tailored treatment approaches to address CPTSD within this population.

\section{References}

Aarts, R., Van Wanrooij, L., Bloemen, E., \& Smid, G. (2019). Expert medico-legal reports: The relationship between levels of consistency 
and judicial outcomes in asylum seekers in the Netherlands. Torture fournal: Fournal on Rehabilitation of Torture Victims and Prevention of Torture, 29. doi:https://doi.org/10.7146/torture. v29i1.111205

Baker, R. (1992). Psychosocial consequences for tortured refugees seeking asylum and refugee status in Europe. In M. Basoglu (Ed.), Torture and its consequences: Current treatment approaches (pp. 83-106). Cambridge: Cambridge University Press.

Basoglu, M. (2006). Little otcome evaluation has been done in torture rehabilitation. British Medical fournal, 2006(333), 1230-1231.

Beiser, M., \& Hou, F. (2001). Language acquisition, unemployment and depressive disorder among Southeast Asian refugees: a 10-year study. Social science $\mathcal{E}$ medicine, 53(10), 1321-1334.

Beltran, R. O., \& Silove, D. (1999). Expert opinions about the ICD-10 category of enduring personality change after catastrophic experience. Comprehensive Psychiatry, 40(5), 396-403. doi:10.1016/s0010-440x(99)90147-5

Ben-Ezra, M., Hyland, P., Karatzias, T., Maercker, A., Hamama-Raz, Y., Lavenda, O., . . . Shevlin, M. (2020). A cross-country psychiatric screening of ICD-11 disorders specifically associated with stress in Kenya, Nigeria and Ghana. Eur $\mathcal{F}$ Psychotraumatol, 11(1), 1720972. doi:10.1080/20 008198.2020.1720972

Cloitre, M., Courtois, C. A., Charuvastra, A., Carapezza, R., Stolbach, B. C., \& Green, B. L. (2011). Treatment of complex PTSD: results of the ISTSS expert clinician survey on best practices. F Trauma Stress, 24(6), 615-627. doi: $10.1002 /$ jts. 20697

Cloitre, M., Shevlin, M., Brewin, C. R., Bisson, J. I., Roberts, N. P., Maercker, A., . . Hyland, P. (2018). The International Trauma Questionnaire: development of a self-report measure of ICD-11 PTSD and complex PTSD. Acta Psychiatr Scand, 138(6), 536-546. doi:10.1111/acps. 12956

Courtois, C. A., \& Ford, J. D. . (2009). Treating complex traumatic stress disorders: An evidence-based guide. New York: NY: Guilford Press.

Crumlish, N., \& Bracken, P. (2011). Mental health and the asylum process. Irish fournal of Psychological Medicine, 28(2), 57-60. doi:10.1017/ S0790966700011447

Crumlish, N., \& O'Rourke, K. (2010). A Systematic Review of Treatments for Post-Traumatic Stress Disorder Among Refugees and Asylum-Seekers. In (Vol. 198, pp. 237-251).

de C Williams, A. C., \& van der Merwe, J. (2013). The psychological impact of torture.
British fournal of Pain, 7(2), 101-106. doi:10.1177/2049463713483596

Forbes, D., Bisson, J. I., Monson, C. M., \& Berliner, L. (2020). Effective treatments for PTSD: Guilford Publications.

Herman, J. (1992a). Complex PTSD: A syndrome in survivors of prolonged and repeated trauma. fournal of traumatic stress, 5(3), 377-391.

Herman, J. (1992b). Trauma and recovery: The aftermath of violence--from domestic abuse to political terror: Hachette UK.

Ibrahim, H., \& Hassan, C. Q. (2017). Post-traumatic Stress Disorder Symptoms Resulting from Torture and Other Traumatic Events among Syrian Kurdish Refugees in Kurdistan Region, Iraq. Frontiers in psychology, 8, 241-241. doi:10.3389/fpsyg.2017.00241

Jaranson, J. M., \& Quiroga, J. (2011). Evaluating the services of torture rehabilitation programmes: history and recommendations. Torture, 21(2), 98-140.

Karatzias, T., Hyland, P., Bradley, A., Cloitre, M., Roberts, N. P., Bisson, J. I., \& Shevlin, M. (2019). Risk factors and comorbidity of ICD11 PTSD and complex PTSD: Findings from a trauma-exposed population based sample of adults in the United Kingdom. Depress Anxiety. doi:10.1002/da.22934

Karatzias, T., Shevlin, M., Fyvie, C., Hyland, P., Efthymiadou, E., Wilson, D., ... Cloitre, $M$. (2016). An initial psychometric assessment of an ICD-11 based measure of PTSD and complex PTSD (ICD-TQ): Evidence of construct validity. f Anxiety Disord, 44, 73-79. doi:10.1016/j. janxdis.2016.10.009

Karatzias, T., Shevlin, M., Fyvie, C., Hyland, P., Efthymiadou, E., Wilson, D., . . . Cloitre, M. (2017). Evidence of distinct profiles of Posttraumatic Stress Disorder (PTSD) and Complex Posttraumatic Stress Disorder (CPTSD) based on the new ICD-11 Trauma Questionnaire (ICD-TQ). F Affect Disord, 207, 181-187. doi:10.1016/j.jad.2016.09.032

Kazlauskas, E., Zelviene, P., Daniunaite, I., Hyland, P., Kvedaraite, M., Shevlin, M., \& Cloitre, M. (2020). The structure of ICD-11 PTSD and Complex PTSD in adolescents exposed to potentially traumatic experiences. Fournal of affective disorders, 265, 169-174. doi:https://doi. org/10.1016/j.jad.2020.01.061

Lie, B. (2002). A 3-year follow-up study of psychosocial functioning and general symptoms in settled refugees. Acta Psychiatr Scand, 106(6), 415-425.

Maercker, A., Brewin, C. R., Bryant, R. A., Cloitre, 
M., van Ommeren, M., Jones, L. M., . . . Reed, G. M. (2013). Diagnosis and classification of disorders specifically associated with stress: proposals for ICD-11. World Psychiatry, 12(3), 198-206. doi:10.1002/wps.20057

Marsella, A. J., Friedman, M. J., Gerrity, E. T., \& Scurfield, R. M. . (1996). Ethnocultural aspects of posttraumatic stress disorder: Issues, research, and clinical applications.

Mollica, R. F., \& Caspi-Yavin, Y. (1991). Measuring torture and torture-related symptoms. 3(4), 581587.

Nickerson, A., Bryant, R. A., Silove, D., \& Steel, Z. (2011). A critical review of psychological treatments of posttraumatic stress disorder in refugees. Clin Psychol Rev, 31(3), 399-417. doi:10.1016/j.cpr.2010.10.004

Nickerson, A., Cloitre, M., Bryant, R. A., Schnyder, U., Morina, N., \& Schick, M. (2016). The factor structure of complex posttraumatic stress disorder in traumatized refugees. Eur $\mathcal{F}$ Psychotraumatol, 7, 33253. doi:10.3402/ejpt. v7.33253

O’Connell, M., Duffy, R., \& Crumlish, N. (2016). Refugees, the asylum system and mental healthcare in Ireland. BfPsych Int, 13(2), 35-37. doi:10.1192/s2056474000001082

OHCHR. (2004). Manual on the Effective Investigation and Documentation of Torture and Other Cruel, Inhuman or Degrading Treatment or Punishment ("Istanbul Protocol”). "Istanbul Protocol”.

Patel, N., Williams, A. C. d. C., \& Kellezi, B. (2018). Reviewing outcomes of psychological interventions with torture survivors: Conceptual, methodological and ethical Issues. Torture fournal, 26(1), 15. doi:10.7146/torture.v26i1.108060

Pérez-Sales, P. (2018). Migration and torture: Building a map of knowledge. Torture fournal, 28(2), 1-14. doi:10.7146/torture.v28i2.106906

Pietrzak, M. (2018). Providing Rehabilitation to Victims of Torture and Other Ill-Treatment UNCAT Implementation Tool. 5/2018.

Shevlin, M., Hyland, P., Vallieres, F., Bisson, J., Makhashvili, N., Javakhishvili, J., . . Roberts, B. (2018). A comparison of DSM-5 and ICD-11 PTSD prevalence, comorbidity and disability: an analysis of the Ukrainian Internally Displaced Person's Mental Health Survey. Acta Psychiatr Scand, 137(2), 138-147. doi:10.1111/acps. 12840

Toar, M., O’Brien, K. K., \& Fahey, T. (2009). Comparison of self-reported health \& healthcare utilisation between asylum seekers and refugees: an observational study. BMC public health, 9(1), 214.

UNCAT. (1984). Convention Against Torture and
Other Cruel Inhuman or Degrading Treatment or Punishment.

UNCERD. (2011). The Policy of Direct Provision: Concluding observations of the Committee on the Elimination of Racial Discrimination. Retrieved from http://www2.ohchr.org/english/bodies/cerd/ docs/co/Ireland_AUV.pdf

UNHCR:Ireland. (2019). Figures at a glance. Retrieved from https://www.unhcr.org/en-ie/

Vaage, A. B., Thomsen, P. H., Silove, D., WentzelLarsen, T., Van Ta, T., \& Hauff, E. (2010). Longterm mental health of Vietnamese refugees in the aftermath of trauma. 196(2), 122-125.

Vallières, F., Ceannt, R., Daccache, F., Abou Daher, R., Sleiman, J., Gilmore, B., . . Hyland, P. (2018). ICD-11 PTSD and complex PTSD amongst Syrian refugees in Lebanon: the factor structure and the clinical utility of the International Trauma Questionnaire. Acta Psychiatr Scand, 138(6), 547-557. doi:10.1111/ acps. 12973

van der Kolk, B. A., Roth, S., Pelcovitz, D., Sunday, S., \& Spinazzola, J. (2005). Disorders of extreme stress: The empirical foundation of a complex adaptation to trauma. F Trauma Stress, 18(5), 389399. doi: $10.1002 /$ jts. 20047

Vang, M. L., Nielsen, S. B., Auning-Hansen, M., \& Elklit, A. (2019). Testing the validity of ICD-11 PTSD and CPTSD among refugees in treatment using latent class analysis. Torture fournal, 29(3), $27-45$.

WHO. (2018). International statistical classification of diseases and related health problems (11th Revision). Retrieved from https://icd.who.int/browse11/I-m/en

Wilson, F. E., Hennessy, E., Dooley, B., Kelly, B. D., \& Ryan, D. A. (2013). Trauma and PTSD rates in an irish psychiatric population: A comparison of native and immigrant samples. Disaster health, 1(2), 74-83. doi:10.4161/dish.27366

\section{Annex: The International Trauma Questionnaire}

The Reference for the measure is: Cloitre, M., Shevlin M., Brewin, C.R., Bisson, J.I., Roberts, N.P., Maercker, A., Karatzias, T., Hyland, P. (in press). The International Trauma Questionnaire: Development of a self-report measure of ICD-11 PTSD and Complex PTSD. Acta Psychiatrica Scandinavica. DOI: 10.1111/acps.12956. ITQ version below is sourced from Cloitre M, Shevlin 
Not at all A little Bit Moderately $\begin{gathered}\text { Quite a } \\ \text { bit }\end{gathered}$ Extremely

1. Having upsetting dreams that replay part of the experience or are clearly related to 0

1

2

3

4

the experience?

2. Having powerful images or memories that sometimes come into your mind in which you feel the experience 0 1 2 3 4 is happening again in the here and now?

3. Avoiding internal reminders of the experience (for example, thoughts, feelings, or physical sensations)?

4. Avoiding external reminders of the experience (for example, people, places, conversations, objects, activities, or situations)?

5. Being "super-alert", watchful, or on guard?

6. Feeling jumpy or easily startled?

$\begin{array}{lllll}0 & 1 & 2 & 3 & 4\end{array}$

In the past month have the above symptoms:

7. Affected your relationships or social life?

8. Affected your work or ability to work?

9. Affected any other important part of your life such as parenting, or school or college work, or other important activities?

M, Brewin CR, Bisson JI, Roberts NP, Maercker A, Karatzias T, Hyland P. The International Trauma Questionnaire: development of a self-report measure of ICD-11 PTSD and complex PTSD. Acta Psychiatr Scand.
2018 Dec;138(6):536-546. doi: 10.1111/ acps.12956. Epub 2018 Sep 3.

\section{Instructions}

Please identify the experience that troubles 


\begin{tabular}{lccccc}
\hline How true is this of you? & $\begin{array}{c}\text { Not at } \\
\text { all }\end{array}$ & $\begin{array}{c}\text { A little } \\
\text { bit }\end{array}$ & Moderately & $\begin{array}{c}\text { Quite } \\
\text { a bit }\end{array}$ & Extremely \\
\hline $\begin{array}{l}\text { 1. When I am upset, it takes me a } \\
\text { long time to calm down. }\end{array}$ & 0 & 1 & 2 & 3 & 4 \\
$\begin{array}{l}\text { 2. I feel numb or emotionally shut } \\
\text { down. }\end{array}$ & 0 & 1 & 2 & 3 & 4 \\
$\begin{array}{l}\text { 3. I feel like a failure. } \\
\text { 4. I feel worthless. }\end{array}$ & 0 & 1 & 2 & 3 & 4 \\
$\begin{array}{l}\text { 5. I feel distant or cut off from } \\
\text { people. }\end{array}$ & 0 & 1 & 2 & 3 & 4 \\
$\begin{array}{l}\text { 6. I find it hard to stay emotionally } \\
\text { close to people. }\end{array}$ & 0 & 1 & 2 & 3 & 4 \\
\hline
\end{tabular}

In the past month, have the above problems in emotions, in beliefs about yourself and in relationships:

7. Created concern or distress about your relationships or social life?

8. Affected your work or ability to work?

9. Affected any other important parts of your life such as parenting, or school or college work, or other important activities?
0

0

0
1

1

1
2

2

2
3

3

4 you most and answer the questions in relation to this experience.

Brief description of experience. When did the experience occur? (circle one)
a. less than 6 months ago
b. 6 to 12 months ago
c. 1 to 5 years ago
d. 5 to 10 years ago
e. 10 to 20 years ago
f. more than 20 years ago

Below are a number of problems that people sometimes report in response to traumatic or stressful life events. Please read each item carefully, then circle one of the numbers to the right to indicate how much you have been bothered by that problem in the past month.

Below are problems or symptoms that people who have had stressful or traumatic events sometimes experience.The questions refer to ways you typically feel, ways you typically think about yourself and ways you typically relate to others. Answer the following thinking about how true each statement is of you. 